\title{
AN OSCILLATION THEOREM FOR SOLUTIONS OF A CLASS OF PARTIAL DIFFERENCE EQUATIONS
}

FULTON KOEHLER AND CHARLES M. BRADEN

In 1908 Hadamard [2] raised the question: if $u$ is the solution of

$$
\left(\frac{\partial^{2}}{\partial x^{2}}+\frac{\partial^{2}}{\partial y^{2}}\right)^{2} u=p
$$

in a region $R$ with $u=\partial u / \partial n=0$ on the boundary of $R$, does the restriction $p \geqq 0$ imply that $u \geqq 0$ ? Duffin, [1], showed that it is possible, when $R$ is an infinite strip, to construct solutions for this problem which oscillate in sign infinitely often, where $p$ is non-negative everywhere and zero outside a finite subset of R. Loewner [4] and Szegö [5] have constructed other examples showing this same kind of behavior. In this paper we consider a finite difference analogue of Duffin's problem, but prove results analogous to his for a general class of partial difference equations, of which the biharmonic equation is a special case.

Let $u(x, y)$ satisfy a linear partial difference equation of the form:

$$
L u(x, y)=\sum_{i=-\nu}^{p} \sum_{j=-m}^{m} c_{i j} u(x+i, y+j)=0
$$

for all integral $(x, y)$ in the strip $S_{N}: 0 \leqq y \leqq N, x \geqq 0$. The boundary values of $u$ for $-m \leqq y<0$ and for $N<y \leqq N+m$ are assumed to be zero, and those for $-\nu \leqq x<0,0 \leqq y \leqq N$ are arbitrary real numbers. The coefficients $c_{i j}$ are assumed to be real constants, and $c_{i j}=c_{i,-j}$. Let

$$
\left|\begin{array}{lll}
c_{\nu, 0} & c_{\nu, 1} & \cdots c_{\nu, N} \\
c_{\nu,-1} & c_{\nu, 0} & \cdots c_{\nu, N-1} \\
\cdot & \cdot & \cdots \\
c_{\nu,-N} & c_{\nu,-N+1} & \cdots \\
c_{\nu, 0}
\end{array}\right| \neq 0
$$

where $c_{i j}=0$ if $|i|>\nu$ or $|j|>m$. Then the solution $u(x, y)$ is uniquely determined by its boundary values and by its values for $0 \leqq x \leqq \nu-1$, $0 \leqq y \leqq N$. These values are assumed to be real, and $u(x, y)$ is then real for all $(x, y)$ in the strip $S_{N}$.

Let $E u(x, y)=u(x+1, y), Q_{j}(E)=\sum_{i=-\nu}^{\nu} c_{i j} E^{i}$, and let the partial

Received by the editors January 8, 1959. 
difference equation (1) be thought of as a system of ordinary difference equations in $u(x, 0), u(x, 1), \cdots, u(x, N)$. We then have, for each integer $y$ in $[0, N]$,

$$
\Delta(E) u(x, y)=0
$$

$x \geqq 0$,

where

$$
\Delta(E)=\left|\begin{array}{cccc}
Q_{0}(E) & Q_{1}(E) & \cdots & Q_{N}(E) \\
Q_{-1}(E) & Q_{0}(E) & \cdots & Q_{N-1}(E) \\
\cdot & & \cdot \\
\cdot & & \cdot \\
Q_{-N}(E) & Q_{-N+1}(E) & \cdots & Q_{0}(E)
\end{array}\right| .
$$

Let the distinct roots of the equation $\Delta(z)=0$ be $z=r_{1}, r_{2}, \cdots, r_{K}$, and let these roots have multiplicities $1+s_{1}, 1+s_{2}, \cdots, 1+s_{K}$ respectively. We shall refer to these roots as the characteristic roots of the operator $L$ for the strip $S_{N}$. Then

$$
u(x, y)=\sum_{\rho=1}^{K} \sum_{\sigma=0}^{S_{\rho}} a_{\rho \sigma} x^{0} r_{\rho}^{x},
$$

where the coefficients $a_{\rho \sigma}$ are functions of $y$.

We shall be particularly concerned with operators $L$ whose characteristic roots are all nonreal and take up first a sufficient condition for an operator to have this property. Let

$$
f(r, \theta)=\sum_{j=-N}^{N} Q_{j}(r) e^{i j \theta}=Q_{0}(r)+2 \sum_{j=1}^{N} Q_{j}(r) \cos j \theta .
$$

THEOREM 1. If, for every real number $r, f(r, \theta)$ is non-negative and not identically zero for $0 \leqq \theta \leqq 2 \pi$, then the characteristic roots of the operator $L$ are all nonreal.

Proof. Let $D(r, \xi)$ be the Toeplitz form corresponding to $f(r, \theta)$,

$$
D(r, \xi)=\frac{1}{2 \pi} \int_{0}^{2 \pi} f(r, \theta)|T(\xi, \theta)|^{2} d \theta=\sum_{i=0}^{N} \sum_{j=0}^{N} Q_{i-j}(r) \xi_{i} \xi_{j},
$$

where $T(\xi, \theta)=\sum_{k=0}^{N} \xi_{k} e^{i k \theta}, \xi_{k}$ real. Then, for any real $r$, this quadratic form is positive definite, and therefore its determinant cannot be zero, i.e.: $\Delta(r) \neq 0$.

THEOREM 2. Let $L_{1}$ and $L_{2}$ be two operators of the form defined by (1), and let $f_{1}(r, \theta)$ and $f_{2}(r, \theta)$ be the corresponding functions defined by (4). Then the function defined by (4) for the operator $L_{1} L_{2}$ is $f_{1} f_{2}$, provided $N$ is sufficiently large. 
Proof. Let

$$
\begin{aligned}
L_{1}(u, y) & =\sum_{j=-m_{1}}^{m_{1}} Q_{j}^{(1)}(E) u(x, y+j), \\
L_{2} u(x, y) & =\sum_{j=-m_{2}}^{m_{2}} Q_{j}^{(2)}(E) u(x, y+j),
\end{aligned}
$$

and let $Q_{j}^{(1)}=0$ for $|j|>m_{1}, Q_{j}^{(2)}=0$ for $|j|>m_{2}$. Then

$$
\begin{aligned}
L_{1} L_{2} u(x, y) & =\sum_{j=-m_{1}}^{m_{1}} Q_{j}^{(1)}(E) \sum_{k=-m_{2}}^{m_{2}} Q_{k}^{(2)}(E) u(x, y+k+j) \\
& =\sum_{l=-\left(m_{1}+m_{2}\right)}^{m_{1}+m_{2}} Q_{l}(E) u(x, y+l)
\end{aligned}
$$

where $Q_{l}(E)=\sum_{j+k=l} Q_{j}^{(1)} Q_{\mathbf{k}}^{(2)}$. Hence, if $N \geqq m_{1}+m_{2}$, the function $f(r, \theta)$ defined by (4) for the operator $L_{1} L_{2}$ is given by

$$
f(r, \theta)=\sum_{l=-\infty}^{\infty} Q_{l}(r) e^{i l \theta}=\sum_{l=-\infty}^{\infty} \sum_{j+k=l} Q_{j}^{(1)} Q_{k}^{(2)} e^{i l \theta}=f_{1} f_{2} .
$$

The usual difference operator, $L^{(H)}$, arising from Laplace's equation is defined by

$$
L^{(H)} u(x, y)=\sum_{j=-1}^{1} Q_{j}^{(H)}(E) u(x, y+j),
$$

where $Q_{0}^{(H)}=E^{-1}-4+E, Q_{1}^{(H)}=Q_{-1}^{(H)}=1$. The usual operator $L^{(B)}$ arising from the biharmonic equation is taken equal to $L^{(H)^{2}}$, and

$$
L^{(B)} u(x, y)=\sum_{j=-2}^{2} Q_{j}^{B}(E) u(x, y+j),
$$

where $Q_{0}^{B}=E^{-2}-8 E^{-1}+20-8 E+E^{2}, \quad Q_{1}^{B}=Q_{-1}^{B}=2 E^{-1}-8+2 E, \quad Q_{2}^{B}$ $=Q_{-2}^{B}=1$.

THEOREM 3. The characteristic roots of the biharmonic operator are nonreal for all values of $N$.

Proof. For $N \geqq 2$, the result follows from Theorems 1 and 2 and the fact that the biharmonic operator is the square of the harmonic operator. For $N=0,1$ the determinants $\Delta(r)$ defined by (2) become

$$
\Delta_{0}(r)=\rho^{2}-8 \rho+18
$$

and

$$
\Delta_{1}(r)=\left(\rho^{2}-8 \rho+18\right)^{2}-(2 \rho-8)^{2}=\left(\rho^{2}-6 \rho+10\right)\left(\rho^{2}-10 \rho+26\right)
$$


respectively, where $\rho=r+1 / r \geqq 2$, and it is obvious that $\Delta_{0}>0, \Delta_{1}>0$ for real $r$.

Let us assume that the characteristic roots of an operator $L$ for the strip $S_{N}$ are all nonreal. For any fixed integer $y$ in $[0, N]$ let any real solution (3) of (1) be written in the form

$$
u(x, y)=\sum_{\alpha=1}^{l} \rho_{\alpha}^{x}\left(q_{\alpha} e^{i x \theta_{\alpha}}+\bar{q}_{\alpha} e^{-i x \theta_{\alpha}}\right),
$$

where $\rho_{1} \geqq \rho_{2} \geqq \cdots \rho_{l}>0$, and where, for each $\alpha, 0<\theta_{\alpha}<\pi$, and $q_{\alpha}$ is a polynomial in $x$ not identically zero. Let $k \geqq 1$ be the integer such that $\rho_{1}=\rho_{2}=\cdots=\rho_{k}$ and either $k=l$ or $\rho_{k}>\rho_{k+1}$. Then

$$
u(x, y)=\rho_{1}^{x}\left[\sum_{\alpha=1}^{k}\left(q_{\alpha} e^{i x \theta_{\alpha}}+\bar{q}_{\alpha} e^{-i x \theta_{\alpha}}\right)+o(1)\right] \quad \text { as } x \rightarrow \infty,
$$

the numbers $\theta_{1}, \theta_{2}, \cdots, \theta_{k}$ being distinct. Let $\mu$ be the maximum degree of the polynomials $q_{\alpha}, 1 \leqq \alpha \leqq k$. Then

$$
u(x, y)=\stackrel{x}{\rho_{1} x^{\mu}}\left[\sum_{\alpha=1}^{k}\left(c_{\alpha} e^{i x \theta_{\alpha}}+\bar{c}_{\alpha} e^{-i x \theta_{\alpha}}\right)+o(1)\right],
$$

where $c_{1}, c_{2}, \cdots, c_{k}$ are constants, not all zero.

THEOREM 4. If the characteristic roots of the operator $L$ in the strip $S_{N}$ are all nonreal, then the solution of (1), for each $y$ in $[0, N]$, will change sign infinitely many times as $x \rightarrow \infty$.

Proof. For any fixed $y$ in $[0, N]$ let $u(x, y)$ be expressed by $(7)$. Let

$$
T(x)=\sum_{\alpha=1}^{k}\left(c_{\alpha} e^{i x \theta_{\alpha}}+\bar{c}_{\alpha} e^{-i x \theta_{\alpha}}\right) .
$$

For any $\epsilon>0$ let $I_{\epsilon}$ be the set of positive integers $\nu$ for which there exist corresponding integers $\nu_{\alpha}$ such that

$$
\left|\nu \theta_{\alpha}-2 \pi \nu_{\alpha}\right|<\epsilon, \quad \alpha=1,2, \cdots, k .
$$

For any $\epsilon>0, I_{\epsilon}$ contains infinitely many members, (see [3, p. 169]), and if $\nu \in I_{\epsilon}$,

$$
|T(x)-T(x+\nu)|<C \epsilon, \quad C=2 \sum_{\alpha=1}^{k}\left|c_{\alpha}\right| .
$$

For any positive integer $A$, let us suppose that there are no sign changes in $T(x)$ for $x \geqq A$, say $T(x) \geqq 0$ for $x \geqq A$. Choose $x_{0} \geqq A$ such that $T\left(x_{0}\right)>0$, and let $\epsilon=(1 / 2 C) T\left(x_{0}\right)$. Then $T(x)>2^{-1} T\left(x_{0}\right)$ for 
$x=x_{0}+\nu, \nu \in I_{\epsilon}$ and $\sum_{x=A}^{M} T(x) \rightarrow \infty$ as $M \rightarrow \infty$. This, however, is a contradiction, since

$$
\left|\sum_{x=A}^{M} T(x)\right| \leqq \frac{2 C}{\left|1-e^{i \theta}\right|}, \quad \theta=\operatorname{Min}_{\alpha}\left\{\theta_{\alpha}\right\},
$$

for all $M$. The assumption $T(x) \leqq 0$ for $x \geqq A$ also leads to a contradiction. Hence, for any $A, T(x)$ must change sign at least once, and consequently an infinite number of times, for $x \geqq A$.

Let $T\left(x_{0}\right)>0, \epsilon=(1 / 2 C) T\left(x_{0}\right)$. Then, from $(7), u\left(x_{0}+\nu, y\right)>0$ for all sufficiently large values of $\nu$ in $I_{\epsilon}$. In the same way we show that there must exist exist infinitely many values of $x$ such that $u(x, y)<0$.

Returning to Hadamard's question stated in the first paragraph, let us assume that $u$ satisfies the partial difference equation $L u=p$ in the doubly infinite strip $0 \leqq y \leqq N$ and that $u=0$ for $-m \leqq y<0$ and $N<y \leqq N+m$. If the characteristic roots of $L$ in the strip $S_{N}$ are nonreal and if $p(x, y)=0$ for $x \geqq 0$, then, by Theorem $4, u(x, y)$ oscillates in sign infinitely often as $x \rightarrow \infty$. This is true regardless of the values of $p(x, y)$ for $x<0$.

\section{REFERENCES}

1. R. J. Duffin, On a question of Hadamard concerning super-biharmonic functions, J. Math. Phys. vol. 27 (1949) pp. 253-258.

2. J. Hadamard, Sur un problème d'analyse relatif a l'equilibre des plaques élastiques encastrées, Mémoires de l'Academie des Sciences de l'Institut de France, vol. 33, no. 4, 1908.

3. G. H. Hardy and E. M. Wright, An introduction to the theory of numbers, Oxford University Press, 1938.

4. C. Loewner, On generation of solutions of the biharmonic equation in the plane by conformal mappings, Pacific J. Math. vol. 3 (1953) pp. 417-436.

5. G. Szegö, Remark on the preceding paper of Charles Loewner, Pacific J. Math. vol. 3 (1953) pp. 437-446.

UnIVERSITY OF MinNESOTA AND

Macalester College 